\title{
Dynamic Connections in Neural Networks
}

Jerome A. Feldman

Computer Science Department, University of Rochester, Rochester, USA

Biol. Cybern. 46, 27-39 (1982)

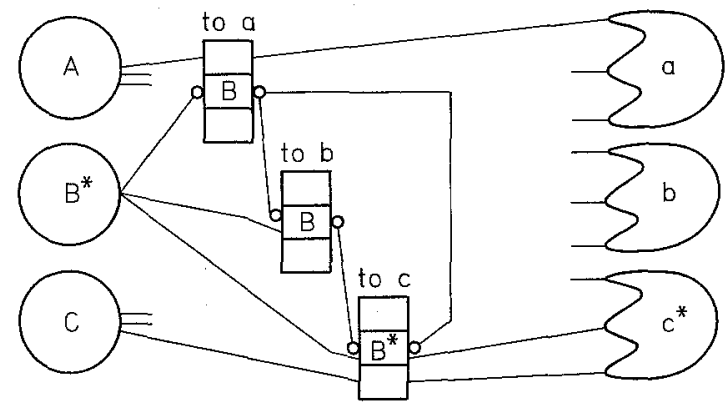

Inter-unit

\begin{tabular}{|c|c|c|c|c|}
\hline & One-end & Dual & Block & - \\
\hline Idle & Low & & Blocked & \\
\hline Low & & & Blocked & Idle \\
\hline High & (Low) & & $x$ & Low \\
\hline Blocked & & $x$ & & Idle \\
\hline
\end{tabular}

End-unit

\begin{tabular}{l|c|c|c}
\multicolumn{3}{c}{ Start } & \multicolumn{2}{c}{$\begin{array}{l}\text { From } \\
\text { inter }\end{array}$} \\
\hline Idle & Low & Low & \\
\hline Low & High & High & Idle \\
\hline High & & (Low) & Low \\
\hline
\end{tabular}

Fig. 4. State and output tables for dynamic connections

On p. 28 , second column, the second formula should be $v \leftarrow$ if $\underline{p}>0 \ldots$ On p. 30 , next to last sentence in the last full paragraph, the final " $B$ " should be "not $B$ ". On p. 33, the formula in the first column should read

$\bar{P}=(1-F)^{B^{K}}$.

On p. 35 , the formula $v \leftarrow 2 p$ should be $v \leftarrow 0.2 p$. Also on p. 36 there is a long formula. The first bracket after the equals is empty. It should be

$\left(\begin{array}{l}d \\ k\end{array}\right)$

which is read " $d$ choose $k$ " and is the number of different combinations of $k$ choices from a total of $d$ alternatives.

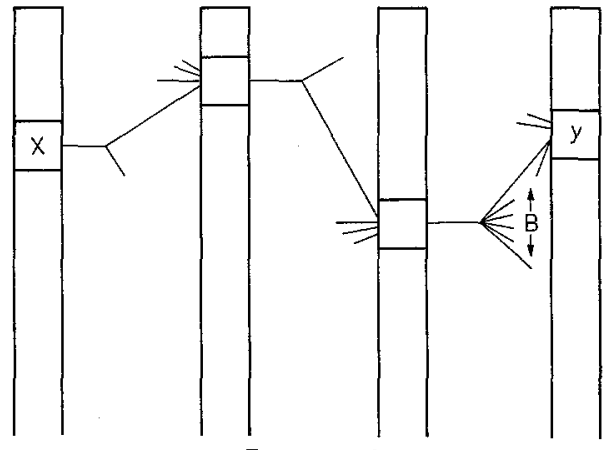

$\bar{P}=(1-F)^{B^{k}}$

$\bar{P}=$ Probability that there is no link from $X$ to $y$

$N=$ Number of Units in a "Layer"

$B=$ Number of Randomly Outgoing Branches/Unit $\approx \sqrt{N}$

$F=B / N$ (Branching Factor)

$K=$ Number of Intermediate Levels ( 2 in diagram above)

$\bar{P}$ for $B=1000$; different numbers of levels and units

\begin{tabular}{llll}
\hline & $N=$ & $10^{7}$ & $10^{8}$ \\
\hline 0 & $10^{6}$ & & 0.99999 \\
1 & 0.999 & 0.9999 & 0.989 \\
2 & 0.367 & 0.905 & $10^{-5}$ \\
\hline
\end{tabular}

Fig. 7. Making a connection

Random networks: $N$ nodes each connected to $\sqrt{N}$ others

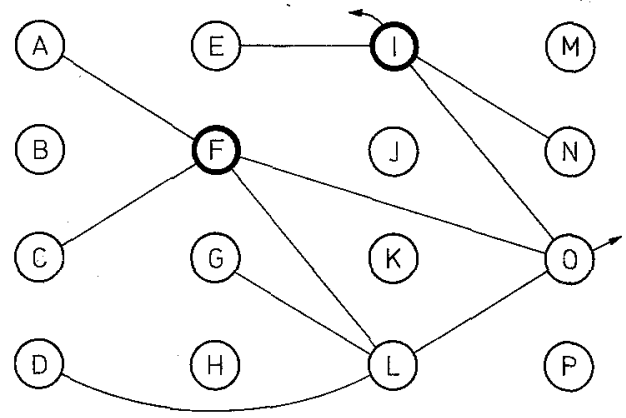

Assume $v=0.2^{*}$ potential; decay is 2

$\begin{array}{rllllllll}T=0 & F & I & G & L & O & A & N & \ldots \\ 1 & 10 & 10 & 0 & 0 & 0 & 0 & 0 & 0 \\ 2 & 10 & 10 & 0 & 2 & 4 & 2 & 2 & \\ 3 & 10 & 10 & 0 & 2.8 & 6 & 2 & 2 & \\ 4 & 10 & 10 & 1 & 4 & 8.6 & 2 & 2 & \\ 5 & 10 & 10 & 1 & 6.3 & 10 & 2 & 2 & \end{array}$

Fig. 8. Random chunking network 